\title{
IMPLICACIONES PEDAGÓGICAS DE UN SOFTWARE DE GEOMETRÍA DINÁMICA EN LA PERCEPCIÓN GEOMÉTRICA DE LAS FUNCIONES TRIGONOMÉTRICAS SENO, COSENO Y TANGENTE
}

\author{
PEDAGOGIC INFLUENCE OF A DYNAMIC GEOMETRY SOFTWARE IN THE GEOMETRIC \\ PERCEPTION OF THE TRIGONOMETRY FUNCTIONS SINE, COSINE AND TANGENT
}

\author{
Jorge Hernán Aristizábal-Zapata*, Ángela María Jiménez-Rojas** y Wilson Andrés Álvarez-Martínez***
}

\section{RESUMEN}

Esta investigación permite evidenciar las implicaciones pedagógicas de un software de geometría dinámica en la percepción geométrica de las funciones trigonométricas seno, coseno y tangente, mediante el modelo de razonamiento de Van Hiele, el cual no solo ayuda a guiar el proceso de enseñanza-aprendizaje en geometría, teniendo como referencia las fases, niveles y características del modelo, sino que coadyuva a evaluar las habilidades cognitivas y comunicativas de los estudiantes. La investigación se demarco desde las políticas y acciones que ha previsto el Ministerio de Educación Nacional (M.E.N.), así como los objetivos del Sistema Nacional de Innovación Educativa, nacida de la Estrategia Nacional de Recursos Educativos Digitales Abiertos (R.E.D.A.). Se trabajó con una población de dieciséis (16) estudiantes, del grado decimo del Colegio Jorge Isaacs, entidad privada, de la ciudad de Armenia (Quindío), bajo el enfoque metodológico cualitativo, evidenciándose al desarrollar la investigación una experiencia significativa al demostrar la pertinencia del abordaje pedagógico y ratificar el desarrollo del nivel de razonamiento a otro nivel de orden superior para el fortalecimiento de la percepción geométrica de las funciones trigonométricas referenciadas.

Palabras clave: Aprendizaje, enseñanza, función trigonométrica, software educativo, secuencia didáctica, modelo de Van Hiele.

\section{ABSTRACT}

In this research pedagogic influence of a dynamic geometry software in the geometric perception of the trigonometry functions sine, cosine and tangent, using Van Hiele's model, which not only helps guide the process of learning in geometry having as reference phases, levels and characteristics of the model, it helps to assess cognitive and communicative skill of students. Research was framed from the policies and actions planned by Ministry of National Education (MEN) and the objectives of the National System of Educational Innovation, created in National Strategy for Digital Open Educational Resources (REDA).

Fecha de recepción: Marzo 04 de 2015 / Fecha de aceptación: Abril 15 de 2015

Tipología: Artículo de Investigación Científica y Tecnológica

Para citar este artículo: Jiménez, R. A., Aristizábal, Z. J., \& Álvarez, M. W. (2015). Implicaciones pedagógicas de un software de geometría dinámica en la percepción geométrica de las funciones trigonométricas seno, coseno y tangente. Praxis. Vol. 11, 30 - 46

\footnotetext{
*Mcs. En Educación, Universidad del Quindío. Colombia. Email: jhaz@uniquindio.edu.co

**Mcs. En Educación y Desarrollo Humano, Universidad del Quindío. Colombia. Email: amjimenez@uniquindio.edu.co

***Lic. Matemáticas, Colegio Jorge Isaacs, Armenia. Colombia. Email: w_andres87@hotmail.com
} 
In this research we worked with a population of sixteen (16) students of Jorge Isaacs School of the Armenia (Q) City under the qualitative methodological approach, highlighting a meaningful experience when the investigation is developed showing the belonging of the pedagogic fact and emphasis the conscious level to other level of superior order to the contribution of the geometric perception of the referenced trigonometric functions.

Keywords: Learning, teaching, trigonometric function, educational software, teaching sequence, Van Hiele's model.

\section{INTRODUCCIÓN}

$\tau$ a investigación surgió de la problemática vivida a diario en los espacios académicos escolares y de distintas investigaciones realizadas, entre otros, por Sánchez (2010): Estrategias didácticas para el aprendizaje de los contenidos de trigonometría empleando las TICs; y Carrillo (2006): Recursos nemotécnicos de las funciones trigonométricas básicas, investigaciones que hicieron explícitas, entre otras cosas: la apatía y la dificultad, por parte de los estudiantes, para aprender las matemáticas. Apatía y dificultad generada por la forma en que las matemáticas son llevadas al aula, a saber: ejercicios rutinarios y descontextualizados, así lo menciona Acosta (2005): Geometría expermiental con Cabri: una praxeología matemática.

Las actividades que se llevan a cabo en el aula, en relación al aprendizaje, el análisis y la comprensión geométrica de las funciones trigonométricas, son limitadoras; ya que, conllevan a que cognitivamente se visualice solo un bosquejo de las gráficas; de tal manera que los estudiantes son inducidos a que retengan dicho esquemas para ser reproducido en el momento requerido. Por tanto, las mencionadas actividades únicamente son el preámbulo de lo que realmente es el proceso de enseñanza-aprendizaje.

Al respecto de ello, Carulla y Gómez (s.f.) destacan la dificultad en la coordinación de los modelos de representación gráfica y algebraica, en los que se hace tácita la presencia de una correlación entre este tipo de representaciones matemáticas, para que se dé el proceso enseñanza-aprendizaje en el aula de clase. Complementario a lo anterior, cabe resaltar el uso de la tecnología en la enseñanza de las matemáticas, para lo cual se reconocen a los software educativos como objeto pedagógico en el mejoramiento de este proceso; dado que el software brinda condiciones apropiadas para el desarrollo del pensamiento lógico-matemático, sin dejar de lado que es un instrumento favorable y práctico que, acompañado de una secuencia de aprendizaje innovadora, mejorará el proceso enseñanza-aprendizaje.

Se pretendió, desde las perspectivas anteriores, y con el sustento que da la didáctica de las matemáticas, entendida ésta como la base sobre la que descansa el proceso de enseñanzaaprendizaje, y que hace asequible que todo el compendio de conocimientos matemáticos se vincule, de manera asertiva, en el entramado del triángulo maestro-saber-estudiante ${ }^{1}$. Por su parte, se entiende por didáctica de las matemáticas una disciplina que se interesa por el estudio de los procesos mediante los cuales los discentes pueden adquirir un saber matemático en una situación escolar específica, es decir, las actividades que tienen como objetivo la enseñanza (Cantoral y Farfán, 2004). La didáctica de las matemáticas, por lo tanto, está orientada a la mejora de los procesos de enseñanza-aprendizaje (Soto, 1993), y al diseñó de instrumentos que coadyuven a la labor docente dentro del aula de clase, al tiempo que motive al estudiante a interesarse por las matemáticas, a lo que Sierra (2001) plantea que:

...los objetos de investigación en Didáctica de la Matemática no se reducen sólo a conocer las circunstancias en las que ocurre la Educación Matemática, sino a determinar los conocimientos, medios y técnicas para mejorar el proceso de Enseñanza-aprendizaje de las Matemáticas (p.176).

1. “...tres sujetos (...) que entran (...) en contacto entre ellos en el momento de la acción didáctica.” (D’Amore, 2006, p.233). 
Tales condiciones han sido tenidas en cuenta en la presente investigación, que tiene como propósito principal evidenciar las implicaciones pedagógicas de un software de geometría dinámica en la percepción geométrica de las funciones trigonométricas seno, coseno y tangente para lo cual se configuró el proceso de enseñanza en una serie de clases estructuradas y secuenciales, lo que generó ambientes propicios de aprendizaje, mediados por guías y, además, se utilizó un software de geometría dinámica ${ }^{2}$, articulado a las disposiciones del Ministerio de Educación Nacional -MEN- y al Ministerio de Tecnologías y de la Información y las Comunicaciones -MINTIC-, con su Documento técnico de especificaciones para el diseño, desarrollo y producción de Unidades Didácticas Digitales, llamado: Desarrollando estrategias de fortalecimiento de la práctica de aula y el aprendizaje de los estudiantes medidas por las TIC; todo el con el fin de identificar y comprender la representación geométrica de las funciones trigonométricas seno, coseno y tangente; claro está, sin olvidar el rigor matemático que brindan las clases habituales en el aula.

En concordancia, para el proceso de enseñanza se elaboraron guías que facilitaron una interpretación matricial de doble entrada, que permitieron analizar la evolución de cada estudiante. Asimismo, posibilitó la validación de cada respuesta dada por los educandos en las guías. Es así como, de manera horizontal, se pudo observar el desenvolvimiento individual, y de manera vertical, se logró notar el tratamiento a cada pregunta dada en la guía y, si se observa la matriz de forma global, se conseguirá percibir el desarrollo general del grupo frente a la guía trabajada. Por otro lado, como modelo pedagógico, se tomó como referente las fases del modelo de Van Hiele, con el horizonte claro de ayudar a los estudiantes a transitar hasta el tercer nivel de razonamiento. Toda vez, que la pedagogía se irgue como el andamiaje posibilitador del proceso enseñanza-aprendizaje; proceso que se tendrá en cuenta a la luz de los razonamientos de Ausubel, Novak y Hanesian (2001).

\footnotetext{
2. Se debe tener en cuenta que dentro de este trabajo no se opta por un software en específico, pues la configuración del proceso de enseñanza-aprendizaje es amplio y permite la labor con diferentes tipos de herramientas informáticas: Geogebra, Cabri, CarMetal, Regla y Compás, entre otros.
}

\section{Modelo de razonamiento de Van Hiele}

Este modelo fue elaborado en la escuela holandesa y explica la evolución del razonamiento geométrico, el cual está dividido en cinco (5) niveles secuenciales, y no independientes, que constituyen un esquema para organizar la enseñanza a lo que Fouz y Donosti (s.f.) afirman que al pasar de nivel se hacen explícitos los conocimientos implícitos que estaban en el nivel anterior, lo que evidencia el aumento del grado de comprensión y dominio del conocimiento. En este sentido, una de las ideas básicas del modelo de Van Hiele, es la siguiente:

...a lo largo del proceso de aprendizaje de la geometría, los estudiantes, pasan por una serie de niveles de razonamiento, que son secuénciales, ordenados y tales que no se puede saltar ninguno. Cada nivel supone la comprensión y utilización de los conceptos geométricos de una manera distinta, lo cual se refleja en una manera diferente de interpretarlos, definirlos, clasificarlos y hacer demostraciones (Rizzolo, s.f., p.4).

Es más, se señala que cualquier persona, ante un nuevo contenido geométrico a aprender se debe tener en cuenta que:

Conocimientos y niveles de razonamiento de los estudiantes no pueden considerarse como realidades absolutamente disociadas. Es necesario pensar el progreso a través de los niveles de razonamiento como un proceso constructivo obligatoriamente ligado al dominio de redes conceptuales cada vez más complejas (Rizzolo, s.f., p. 20).

Igualmente, Van Hiele (1990) concreta que:

...uno ha alcanzado determinado nivel de pensamiento cuando una nueva ordenación mental respecto de ciertas operaciones le permite aplicarlas a nuevos objetos. No se puede llegar a estos niveles con el estudio; sin embargo, el profesor puede, mediante una selección apropiada de tareas, crear una situación ideal para que el alumno alcance un nivel de pensamiento superior. Se puede afirmar además que la consecución de un nivel superior aumenta considerablemente el potencial del alumno... (p.88). 


\section{Figure 1}

Idea básica del razonamiento de Van Hiele.

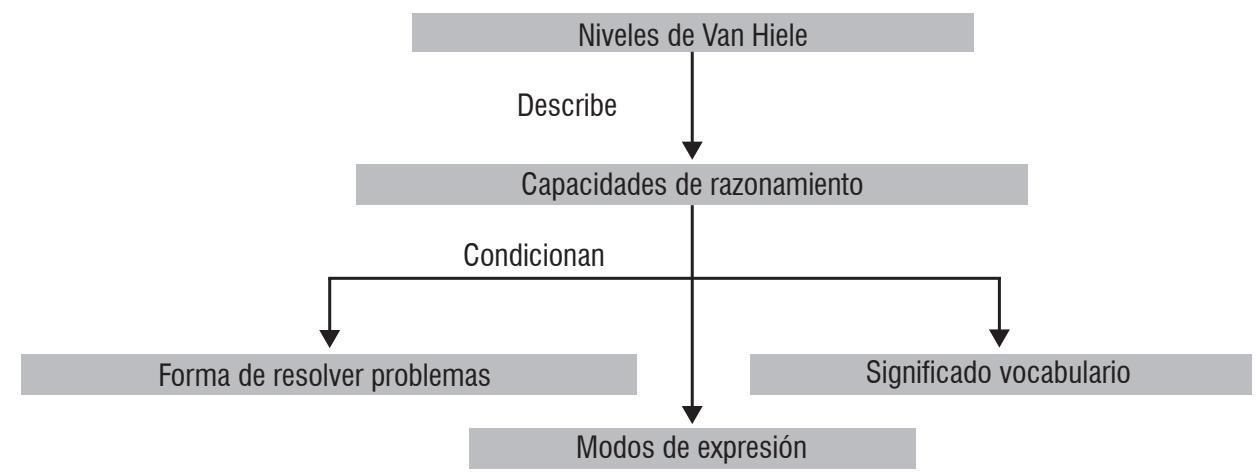

.Fuente: Rizzolo (s.f.).

\section{Niveles de Van Hiele}

Diversos autores enuncian los niveles cero (0) al cuatro (4) y otros del uno (1) al cinco (5); lo que hace tácito que no hay unanimidad en esta numeración. Por lo que, para no crear ambigüedad en esta numeración, el presente artículo se acoge a la descripción que dan los autores Fouz (s.f.) en la siguiente forma: "Nivel 1: visualización o reconocimiento Nivel 2: análisis Nivel 3: ordenación o clasificación Nivel 4: deducción formal Nivel 5: rigor” (p.68). Es significativo recalcar que, en ciertos momentos de la aplicación de contenidos, es normal que un estudiante se encuentre en un nivel diferente al de sus compañeros, pero eso no implica que no esté haciendo bien su camino por los niveles.

\section{Características del modelo}

En este sentido, cabe tener en cuenta lo que apunta Fouz y Donosti (s.f.) al internarse por las características del modelo aquí referenciado, por lo que ellos hablan de la primera característica:

...hablamos de "secuenciación", algo que, visto o explicado hasta ahora, no necesita más explicación, de "jerarquización" esto es, los niveles tienen un orden que no se puede alterar, lo cual es obvio visto también lo anterior y los niveles "son recursivos". Esta última idea es importante y conviene explicarla y concretarla un poco más. Esta característica nos indica que "lo que es implícito en un nivel se convierte en explícito en el siguiente nivel" [comillas y cursivas en texto fuente] (p.70).

Continúa Fouz y Donosti (s.f.) con la segunda característica del modelo Van Hiele el cual es entendido como:

... "el lenguaje" específico para cada nivel. La progresión en y entre los niveles va muy unida a la mejora del lenguaje matemático necesario en el 
aprendizaje. No sé trata sólo de adquirir conocimientos matemáticos sino también mejoras y ampliar las capacidades referidas al lenguaje necesario en cada nivel [comillas y cursivas en texto fuente] (p.71).

Asimismo, el tercer componente de este modelo es descrito por Fouz y Donosti (s.f.) como:

La tercera idea es si el aprendizaje y, por tanto, el paso de nivel se hace de una manera "continua o discreta". La idea, eterno dilema, es si el salto es repentino o se hace de forma gradual. Nos parece lógico pensar que se hace de forma continua mediante pequeños saltos que conexos que nos darán el paso final de nivel. Esto está más de acuerdo con las teorías cognitivas modernas del aprendizaje que señalan cómo creamos esquemas significativos de pensamiento, mejores pero cercanos a los que teníamos, que se interconectan entre sí y que, a su vez, podemos reemplazar por otros nuevas más sencillos y prácticos que los anteriores. Para construir o mejorar estos esquemas tiene mucha importancia la interacción alumno/a-profesor/a [comillas y cursivas en texto fuente] (p.71).

\section{Fases del modelo}

Las fases del modelo de Van Hiele están en correlación directa con el binomio estudiante-docente, en el que el educando cobra preponderancia, por cuanto

Van Hiele caracteriza el aprendizaje como resultado de la acumulación de la cantidad suficiente de experiencias adecuadas; por lo tanto, existe la posibilidad de alcanzar niveles más altos de razonamiento fuera de la enseñanza escolar si se consiguen las experiencias apropiadas. No obstante, esas experiencias, aunque existen y no deben despreciarse, generalmente no son suficientes para producir un desarrollo de la capacidad de razonamiento completo y rápido, por lo que la misión de la educación matemática escolar es proporcionar experiencias adicionales, bien organizadas, para que sean los más útiles posibles. A lo largo de estas fases, el docente debe procurar que sus alumno construyan la red mental de relaciones del nivel de razonamiento al que deben acceder, creando primero los vértices de la red, y después las conexiones entre ellos. Dicho de otra manera es necesario conseguir en primer lugar, que los estudiantes adquieran de manera comprensiva, los conocimientos básicos necesarios, (nuevos conceptos, propiedades, vocabulario...) con los que tendrán que trabajar, para después centrar su actividad, en aprender a utilizarlos y combinarlos (Rizzolo, s.f., p.14).

En consideración que las fases de aprendizaje que representan el componente descriptivo de la teoría, suponen una recomendación a los profesores sobre cómo pueden organizar la actividad en sus clases, para que los estudiantes sean capaces de acceder al nivel de razonamiento inmediatamente superior al que tienen actualmente (Gutiérrez y Jaime, 1998). En concomitancia, las fases que se ligan dentro del modelo de Van Hiele son cinco (5): "Fase 1: preguntas/información. Fase 2: orientación dirigida. Fase 3: explicación (explicitación). Fase 4: orientación libre. Fase 5: integración” Fouz y Donosti (s.f., p.72).

\section{Abordaje pedagógico aplicado al modelo de Van Hile}

La pedagogía es entendida como un conjunto o vademécum de reflexiones sobre la educación, en otras palabras, la pedagogía es la capacidad perenne de reflexionar en torno a la acción educativa; esta reflexión amén de ser fáctica, es prominentemente crítica. De tal suerte, que la pedagogía es la llamada a poner sobre el telón paradigmas educativos que permitan validar o refutar las prácticas educacionales y, por lo mismo, proyectar nuevas realidades propulsoras de trasformaciones sociales (Villalobos, 2006). De lo anterior, la pedagogía es un insumo que coadyuva para que el modelo de Van Hile sea aprehendido de forma significativa por los estudiantes dentro de su proceso enseñanzaaprendizaje. Antes de seguir, es necesario escribir que el aprendizaje significativo es el parapeto posibilitador para que el modelo de Van Hile 
llegar de forma asertiva y real al educando. En este orden de ideas, Ballester (2002) menciona, en referencia al aprendizaje:

...es construcción de conocimiento donde unas piezas encajan con las otras en un todo coherente. Por lo tanto, para que se produzca un auténtico aprendizaje, es decir un aprendizaje a largo plazo y que no sea fácilmente sometido al olvido, es necesario conectar la estrategia didáctica del profesor con las ideas previas del alumnado y presentar la información de manera coherente y no arbitraria, "construyendo", de manera sólida, los conceptos, interconectados los unos con los otros en forma de res de conocimiento (p.16).

\section{Figure 2}

Fases del Modelo de Van Hiele

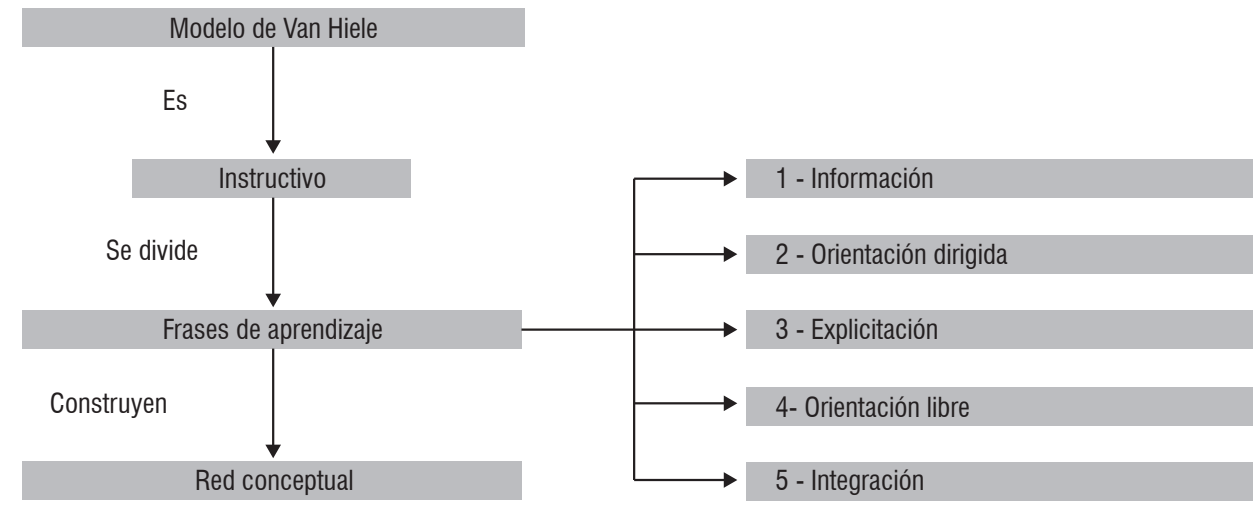

Fuente: Rizzolo (s.f.).

Ausubel, Novak y Hanesian (2001) diferencian, claramente, entre el aprendizaje memorístico y el significativo: en el primero el estudiante introyecta la información y la ajusta a sus esquemas mentales, con el fin de movilizarse con dicha información de manera lineal y textual; Pozo (1994) por su parte, escribe: "El aprendizaje memorístico o por repetición es aquel en el que los contenidos están relacionados entre sí de un modo arbitrario, es decir careciendo de todo significado para la persona que aprende” (p. 212).

El segundo aprendizaje posibilita que en el estudiante se evidencie un desequilibrio cognitivo entre sus conocimientos previos y experiencias, esto debido al introyectar la información y realizar un ajuste, fortalecimiento o transformación total o parcial de los esquemas con los cuales se moviliza, lo que conlleva, dicho conocimiento, a una relación no arbitraria ni textual. Todo lo contrario, permitiéndole al educando movilizarse con el mencionado conocimiento en cualquier escenario; toda vez, que se convierte en parte de su repertorio cognitivo, lo que evidencia su utilidad y practica; es decir, Ausubel, Novak y Hanesian (2001) enuncian: "El aprendizaje significativo comprende de la adquisición de nuevos significados y, a la inversa, estos son producto del aprendizaje significativo. Esto es, el surgimiento de nuevos significados en el alumno refleja la consumación de un proceso de aprendizaje significativo" (p.48). 
El aprendizaje significativo, por tal razón, le permite al estudiante, de forma consciente, relacionar una información nueva con la estructura cognitiva, esto está correlacionado directamente con el modelo de Van Hile, puesto que al ahondar por los diferentes niveles y fases del modelo se fortalece el aprendizaje significativo en la percepción geométrica de las funciones trigonométricas.

\section{Diseño metodológico}

Para investigar sobre las implicaciones pedagógicas de un software de geometría dinámica, en la percepción geométrica de las funciones trigonométricas seno, coseno y tangente, se utilizó el tipo de investigación cualitativa. (Hernández, Fernández y Baptista, 2010) En la misma medida el enfoque del que se echó mano es descriptivo. Por otra parte, la población sujeto de estudio estuvo conformada por dieciséis (16) estudiantes de décimo grado de educación básica secundaria, del colegio privado Jorge Isaacs. Asimismo, se trabajó con una intensidad de cinco (5) horas semanales, en las que cada educando desarrolló las actividades de manera individual en los computadores, lo que le permitió tener contacto directo con el software dinámico.

En el proceso de investigación cualitativa, la recolección y el análisis de datos ocurren prácticamente en paralelo; además, el análisis no es estándar, puesto que cada estudio requiere de un esquema. De tal forma que por validación de la investigación se tomará esencialmente como interna, basada en la confrontación entre el análisis de la prueba preliminar y la prueba posterior, sin dejar de lado las observaciones vistas en el transcurso de proceso investigativo. En concomitancia, este diseño es denominado "diseño preliminar posterior con un grupo", el mismo tiene la siguiente estructura:

$$
G P_{1} T P_{2}
$$

G: es el grupo.

$\mathrm{P}_{1}$ : Prueba Preliminar (Antes).

$\mathrm{P}_{2}$ : Prueba Posterior (después).

T: Tratamiento.
Por otra parte, es necesario tener en cuenta lo que postulan Hernández, Fernández y Baptista (2010), en relación a la recolección de datos, que llevan, ineluctablemente, a la objetividad que este trabajo perseguía:

En la recolección de datos, la acción esencial consiste en que recibimos datos no estructurados, (...) Los datos son muy variados, pero en esencia consisten en narraciones de los participantes: a) visuales (fotografías, videos, pinturas, entre otros), b) auditivas (grabaciones), c) textos escritos (documentos, cartas, etc.) y d ) expresiones verbales y no verbales (como respuestas orales y gestos en una entrevista o grupo de enfoque), además de las narraciones del investigador (anotaciones o grabaciones en la bitácora de campo, ya sea una libreta o un dispositivo electrónico) (p.439).

Luego de recibidos los datos no estructurados, se continuó con su organización. Lo que llevó a un ordenamiento de todo el vademécum de la información recogida:

- Darle estructura a los datos (...), lo cual implica organizar las unidades, las categorías, los temas y los patrones...

- Describir las experiencias de las personas estudiadas bajo su óptica, en su lenguaje y con sus expresiones...

- Comprender en profundidad el contexto que rodea los datos...

- Interpretar y evaluar unidades, categorías, temas y patrones....

- Explicar ambientes, situaciones, hechos, fenómenos

- Reconstruir historias...

- Encontrar sentido a los datos en el marco del planteamiento del problema...

- Relacionar los resultados del análisis con la teoría fundamentada o construir teorías..... (Hernández, Fernández y Baptista, 2010, p.440)

El análisis, de lo que se sigue, es un proceso ecléctico y sistemático, más no rígido ni mecánico; y como cualquier tipo de análisis, el cualitativo es contextual, e involucra el estudio 
de cada pieza de los datos en sí misma, en el que el investigador analiza cada dato (que por sí mismo tiene un valor), deduce similitudes y diferencias con otros datos en el cotejo entre la prueba preliminar y la prueba posterior.

\section{RESULTADOS}

\section{Recolección de datos}

En la búsqueda del estado previo de los estudiantes, se aplicó una prueba preliminar, que consistió en dieciocho (18) preguntas, entre selección múltiple, respuesta libre, graficar, parear (gráfico, nombre, función) y concluir a partir de datos o gráficas. Lo anterior, permitió obtener acceso al lenguaje utilizado, para determinar en qué nivel de razonamiento se encontraban (Fase 1. Información), la cual le permitió al discente: un contacto con el objeto de estudio, y al docente: identificar los conocimientos previos del estudiante, esta fase, igualmente, posibilitó identificar los procesos cognitivos del educando en torno a las funciones trigonométricas. En concordancia, al buscar el mejoramiento en los procesos enseñanza-aprendizaje, relacionados con la representación geométrica de las funciones trigonométricas en los estudiantes, se construyeron una serie de ambientes pedagógicos, seleccionados cuidadosamente, organizados y basados en la manipulación de un software de geometría dinámica, que brindara las herramientas adecuadas para la comprensión del concepto matemático (Fase 2. Orientación Dirigida), en esta fase se evidenció una planeación y mediación pedagógica, por medio de las clases, guías y el uso del software, lo que permitió que los estudiantes realizaran ajustes y resolvieran problemas referidos a las funciones trigonométricas seno, coseno y tangente.

\section{Procedimientos de validación}

Para dar validez a las respuestas obtenidas, se realizó una interpretación matricial de doble entrada, que permitió analizar la evolución de cada estudiante, lo que además facilitó la validación de cada respuesta dada por los estudiantes en todas las guías. Por su parte, de manera horizontal, se pudo observar el desenvolvimiento individual. De otra parte, de manera vertical se logró notar el tratamiento a cada pregunta dada en la guía y, si se mira la matriz de manera global, se hace diáfano percibir el desarrollo general del grupo frente a la guía trabajada. Así mismo, al observar la matriz de doble entrada, referente a la prueba preliminar, se evidencia que algunos estudiantes presentaron muchas falencias en torno al lenguaje utilizado (Fase 3. Explicitación). Esta fase es determinante, toda vez que invita al educando a expresar en lenguaje, sea este escrito $u$ oral, los conceptos matemáticos técnicos fundamentales para la adquisición del aprendizaje. Esto se explicita en las respuestas de algunos estudiantes, como bien lo muestran la figura 3, 4 y 5: 


\section{Figure 3}

Respuesta del Estudiante 9 al ítem 1 de la Guía 1

1. Defina con sus palabras que es una función.
UNA FUNCiON ES UNA MAgNitud O CANtidad

Fuente: Elaboración propia.

\section{Figure 4}

Respuesta del Estudiante 14 al ítem 1 de la Guía 1

1. Defina con sus palabras que es una función.
es La Racion entre un triangota rectangule con
respecto al angulo dado

Fuente: Elaboración propia.

\section{Figure 5}

Respuesta del Estudiante 16 al ítem 1 de la Guía 1

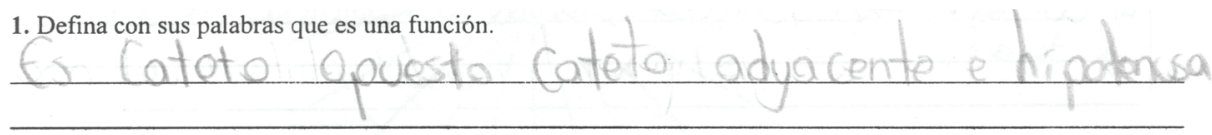

Fuente: Elaboración propia.

A través de la matriz de doble entrada, se puede observar de manera general, un buen desarrollo y comprensión de ángulos sexagesimales; igualmente, en las respuestas coherentes, dadas por los estudiantes, se nota la facilidad que brinda el software para identificar los ángulos, sus características y sus representaciones gráficas (Fase 4. Orientación libre). En esta fase se percibe, en los educandos una modificación en sus esquemas, en la medida en que se refleja un equilibrio cognitivo que hace asequible el establecimiento de relaciones en el uso de los conocimientos adquiridos, el lenguaje técnico, etc., todo con autonomía del educando, para aplicarlos a situaciones nuevas y diferentes.

En relación a esta guía, fue diseñada con el objetivo de identificar, interpretar, medir y verificar equivalencias entre ángulos dados en el sistema sexagesimal y entre ángulos dados en radianes; por lo mismo, se parte de la amplitud de los ángulos que forma al deslizar un punto sobre la circunferencia unitaria, y un punto que se mueve sobre la longitud de una circunferencia expandida sobre el eje $\mathrm{x}$, con las equivalencias en radianes. Por ello, se hace notorio que la herramienta de geometría dinámica facilita la comprensión entre la equivalencia de dos sistemas para medir ángulos, ya que la cantidad de respuestas malas son pocas y dispersas. 


\section{Figure 6}

Respuesta del Estudiante 7 al ítem 10 de la Guía 3

10. Escriba con sus propias palabras que es un ángulo, que tipos de ángulos hay y represéntelos gráficamente.

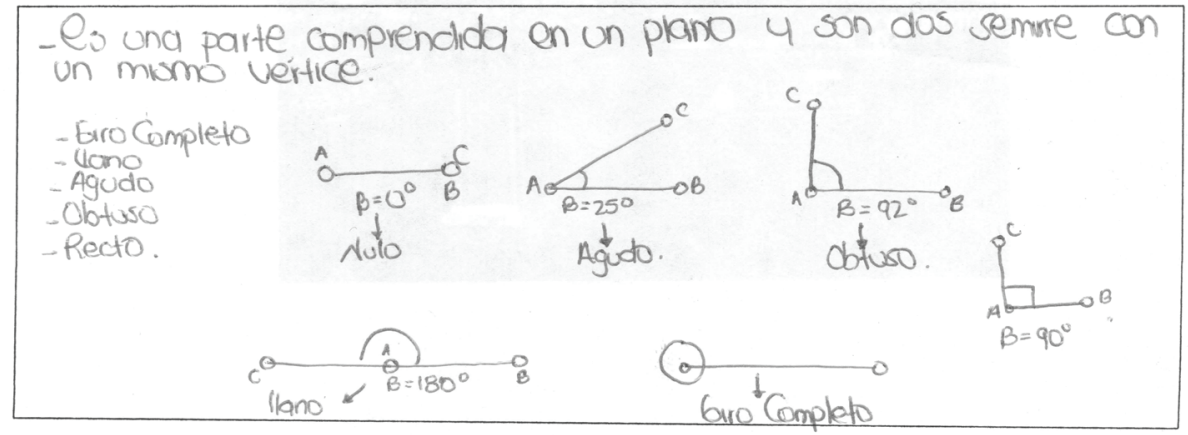

Fuente: Elaboración propia.

\section{Figure 7}

Respuesta del Estudiante al ítem 1-2 de la Guía 4

1. Cuando $\mathrm{A}^{\prime}$ empieza a moverse y coincide con el eje $\mathrm{x}$ por primera vez. ¿Cuántos grados mide $\alpha$ y en cuantos radianes esta $P$ ?

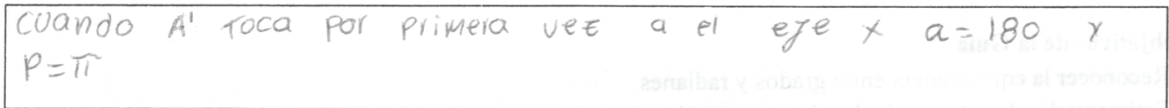

2. Responda con fracciones de $\pi$ : ¿Cuántos radianes mide un ángulo de $90^{\circ}$ ? ¿Y uno de $180^{\circ}$ ? ¿Y uno de $270^{\circ}$ ?

$90^{\circ}=\pi / 2$
$180^{\circ}=\pi$
$270^{\circ}=3 \pi / 2$$\quad$ Estos grados equivalen asi en pi $(\pi)$

Fuente: Elaboración propia.

Posteriormente, cuando se le solicitó a los estudiantes analizar la relación entre la circunferencia y las funciones trigonométricas seno, coseno y tangente; trabajando con alturas y tiempos en un contexto real, por medio de un software en línea (Noria), en el cual se percibían dos personajes: Marta y Pepe que viajaban en dos barquillas diferentes de una noria (rueda) de feria. Se trataba de una noria un poco especial, ya que permitía modificar la velocidad de giro y su radio. Cabe señalar, que al iniciar el viaje los dos personajes están situados a la misma altura del suelo.

Los ítems de la guía estaban diseñados, inicialmente, para que el estudiante describiera, con sus palabras, y realizara un bosquejo de cómo creería que sería el recorrido de Marta y Pepe de manera progresiva con referencia a sus posiciones en la noria. Se denota que la herramienta facilitó este tipo de ejercicios; pues ya se tiene el recorrido y, posteriormente, se enseña la gráfica para que verifiquen y analicen sus respuestas. De tal forma, que las preguntas siguientes van dirigidas a que el estudiante visualice cómo es el comportamiento del recorrido de las posiciones de Marta y Pepe al hacer variar la altura, mientras la noria da vueltas; esto con el propósito que identifiquen los máximos y los mínimos que alcanza cada uno (Fase 
5. Integración). En esta fase se devela una fusión de los conocimientos previos con los nuevos lo que denota una nueva red conceptual, de tal manera que en esta fase se tiene una visión holística y trasversal del conocimiento.

\section{Figure 8}

Respuesta del Estudiante 10 al ítem 12 de la Guía 6

12. Construye la gráfica de la altura sobre el suelo de Marta en función del tiempo si el periodo es 50 segundos y el radio de la noria mide 12,5 metros. Considera que, al igual que en los casos anteriores, la noria está girando durante 5 minutos. Construye sobre los mismos ejes la gráfica de la altura de Pepe.

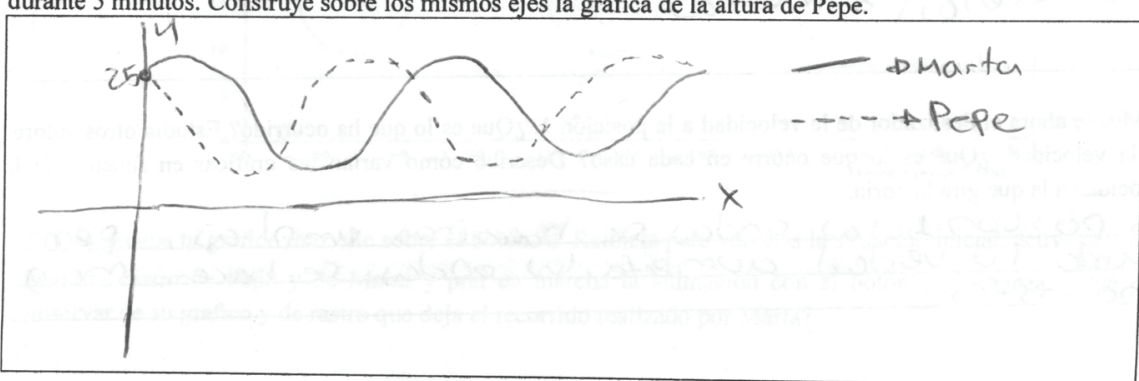

Fuente: Elaboración propia.

En cuanto a los niveles:

Nivel 1. Al relacionar los ángulos se observa la particularidad que en el ítem uno (1), a la gran mayoría de los estudiantes se les dificultó identificar ángulos en los contextos cotidianos, únicamente dos educandos dieron respuestas acordes a lo que se les solicitó señalar; puesto que se les pidió que miraran la imagen de un puente colgante; acto seguido que mencionaran la cantidad de ángulos que allí encontraban. De tal forma que, gracias a las respuestas de ellos, se dejó ver la falencia que tienen para visualizar ángulos en su vida diaria.

\section{Figure 9}

Gráfica dada para identifica ángulos en contexto. Puente Golden Gate ubicado en la ciudad de San Francisco, Estados Unidos.

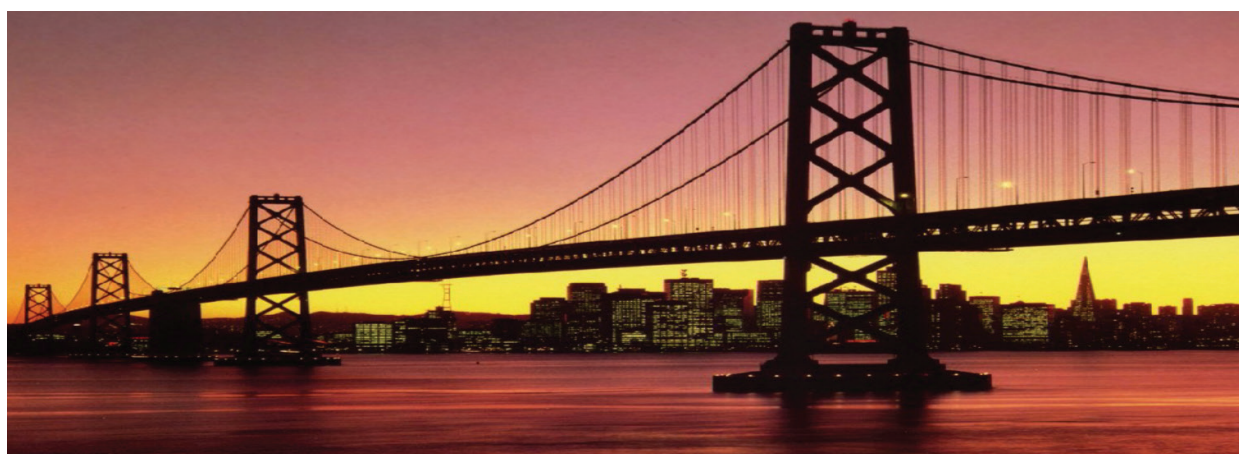

Fuente: http://vicoslan.blogspot.com/2013/05/puente-de-san-francisco.html 


\section{Figure 10}

Respuesta del Estudiante 3 al ítem 1 de la Guía 3

1. ¿Cuántos ángulos puedes señalar?

$$
28 \text { angubs }
$$

Fuente: Elaboración propia.

\section{Figure 11}

Respuesta del Estudiante 13 al ítem 1 de la Guía 3

1. ¿Cuántos ángulos puedes señalar? aproximadamente mas de 26 Pues se van formando entie los 2 postep
del puente ta carleteva

Fuente: Elaboración propia.

Nivel 2. Cuando se le realizaron cambios a las variables más influyentes, como la velocidad en la Noria, los estudiantes expresan, de manera acorde, lo que sucedía con los recorridos de las gráficas que tenían menos puntos de corte; al mismo tiempo, se hizo variar el tiempo y la velocidad del recorrido, de allí, la matriz permitió que se denotara la percepción que tuvieron con respecto al concepto de periodo.

\section{Figure 12}

Respuesta del Estudiante 11 al ítem 10 de la Guía 6

10. Vamos a llamar periodo al tiempo que tarda la noria en dar una vuelta. ¿Cuál es el periodo cuando la velocidad es 1 ? ¿Y si es 2 ? Escribe el periodo que corresponde a cada una de las velocidades. ¿Qué tiene que ver el periodo con la forma de las gráficas que vas obteniendo?

* Cuanda la vebadad esta eny sectemora 300 segndos para hacer una vuel ta Nies la velocidad en 2 sedemora 150 segundos para dar welta completa Cuands el poriodo es menos la grafica nos muestra vuettas la vebcidad podria Ser menos rapida dependiendo de el periodo

Fuente: Elaboración propia.

Nivel 3. La capacidad que adquirieron los estudiantes para relacionar una serie de representaciones geométricas que han sufrido cambios en su amplitud, en su periodo y en sus traslaciones con sus respectivas expresiones algebraicas fue significativa; dado que solo cuatro educandos tuvieron dificultad en este tipo de ejercicio. Es así, que se puede decir que la mayoría de los estudiantes lograron un nivel más avanzado en la comprensión de las representaciones geométricas de las funciones trigonométricas. 


\section{Figure 13}

\section{Respuesta del Estudiante 1 al ítem 5 de la Guía 15}

5. A continuación se describen algunas gráficas de las funciones trigonométricas, utilice Geogebra y relaciónelas con sus respectivas representaciones algebraicas.

a. La función coseno trasladada 3 unidades hacia arriba, y su periodo es 4 .

b. La función seno trasladada 4 unidades hacia abajo, su amplitud es 3 y su periodo aumentado en 5 .

c. La amplitud de la función tangente es 2 y trasladadas 2 unidades a la derecha.

d. La función seno ampliada -2 unidades, trasladad 3 unidades hacia la izquierda, 3 de periodo y 2 hacia abajo.

e. La función coseno trasladada 5 unidades hacia abajo, con un periodo de 6 y ampliada -2 .

f. La función tangente con periodo de -4 , trasladada 2 unidades hacia abajo y 3 hacia la izquierda.

$$
\begin{array}{ll}
\frac{C}{e} \mathrm{r}(\mathrm{x})=2 \tan (1 x-2) & \mathrm{g} p(\mathrm{x})=1 \cos (4 x)+3 \\
\hline \mathrm{b} g(\mathrm{x})=-2 \cos (6 x)-5 & \mathrm{f}(\mathrm{x})=1 \tan (-4 x+3)-2 \\
\mathrm{~g} g(\mathrm{x})=3 \sin (5 x)-4 &
\end{array}
$$

Fuente: Elaboración propia.

\section{Figure 14}

Respuesta del Estudiante 12 a Ítem 6 de la Guía 15

6. Observe cada gráfica y relaciónela con su respectiva expresión; según su amplitud, su periodo y sus traslaciones.

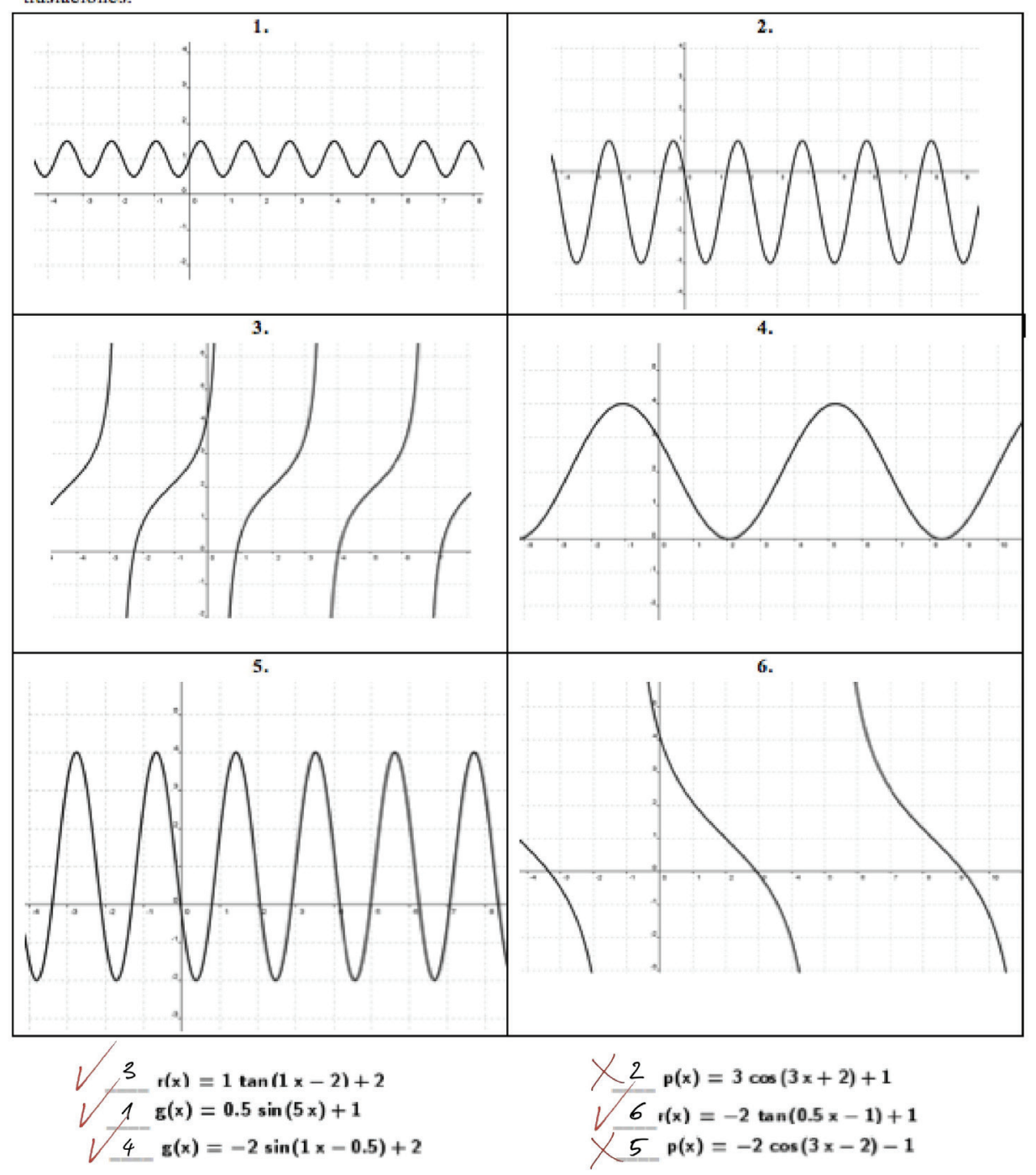

Fuente: Elaboración propia. 


\section{DISCUSIÓN}

Para verificar las implicaciones del software de geometría dinámica, en el proceso de enseñanzaaprendizaje de las funciones trigonométricas a través del abordaje pedagógico planteado, se examinó cada uno de los ítems en la prueba preliminar y en la prueba posterior, haciéndose necesaria la confrontación entre los ítems planteados. De tal suerte que se hizo imperante precisar que la prueba preliminar era una actividad prevista para examinar y recolectar información previa sobre la noción del tema que tienen los estudiantes. La prueba mencionada constaba de dieciocho (18) ítems con preguntas abiertas, en el que se indagaba por el concepto de ángulos y sus características, conversiones y equivalencia entre grados sexagesimales y radianes. También sobre la definición, representación algebraica y gráfica de las funciones, con algunas particularidades. De igual forma, se presentó el triángulo rectángulo con sus respectivas razones trigonométricas, para que el estudiante describiera qué relación distinguía, sobre cómo percibía posibles trayectorias y que descubriera variables que influencian cambios en dichos recorridos. En cuanto a la prueba posterior, se trataba de una actividad diseñada para verificar el nivel de comprensión que alcanzó cada estudiante, la cual constaba de dieciocho (18) ítems, igual que la prueba a cotejar. Se debe escribir, que en esta prueba -así como en la preliminar- se indaga por las nociones y particularidades nuevas de ángulos, las equivalencias, conversiones entre grados sexagesimales y radianes. De igual manera, sobre la relación entre el triángulo rectángulo y las respectivas razones trigonométricas, así como por la visualización de trayectorias, la representación geométrica y algebraica de funciones, con sus respectivos dominios, rangos, amplitudes, periodos y traslaciones.

Por lo anterior, fue preciso y oportuno esclarecer que los ítems de estas pruebas no son los mismos, ni tienen el mismo orden en la temática a verificar. Toda vez, que no se consideró prudente por el nivel que ejerce cada pregunta y cada guía en relación a nuevos conocimientos y nociones. Cabe acotar que en el emparejamiento proporcionado a los ítems, de la prueba preliminar y la prueba posterior, se menciona el orden que se determinó para percibir el progreso de cada estudiante en los temas señalados. A continuación, se muestra la relación que existe entre los ítems de la prueba preliminar y la prueba posterior, y se expone el nivel y la fase a la que apunta cada ítem frente al modelo planteado por Van Hiele en la Tabla 1.

\section{Tabla 1}

Emparejamiento Ítems Prueba Preliminar y Prueba Posterior

\begin{tabular}{|c|c|c|}
\hline $\begin{array}{c}\text { Prueba } \\
\text { Preliminar }\end{array}$ & $\begin{array}{c}\text { Prueba } \\
\text { Posterior }\end{array}$ & $\begin{array}{c}\text { Nivel de } \\
\text { Razonamiento }\end{array}$ \\
\hline Ítem 1 & Ítem 6 & N1-N2 \\
\hline Ítem 2 & Ítem 10 & N1-N2 \\
\hline Ítem 3 & Ítem 12-14 & N1-N2-N3 \\
\hline Ítem 4 & Ítem 1 & N1-N2 \\
\hline Ítem 5 & Ítem 2 & N1-N2-N3 \\
\hline Ítem 6 & Ítem 3 & N1-N2-N3 \\
\hline Ítem 7 & Ítem 4 & N2-N3-N4 \\
\hline Ítem 8 & Ítem 4 & N3-N4 \\
\hline Ítem 9 & Ítem 12-14 & N1-N2 \\
\hline Ítem 10 & Ítem 11 & N1-N2-N3 \\
\hline Ítem 11 & Ítem 13-14 & N1-N2-N3 \\
\hline Ítem 12 & Ítem 4-5 & N1-N2-N3-N4 \\
\hline Ítem 13 & Ítem 7-8-9 & N1-N2 \\
Ítem 14 & Ítem 7-8-9 & N1-N2-N3 \\
\hline Ítem 15 & Ítem 7-8-9 & N1-N2-N3 \\
\hline Ítem 16 & Ítem 15-16 & N1-N2-N3 \\
\hline Ítem 17 & Ítem 17-18 & N1-N2-N3 \\
\hline Ítem 18 & Ítem 17-18 & N1-N2-N3 \\
\hline
\end{tabular}

Fuente: Elaboración propia.

Con respecto a la confrontación de resultados de la prueba preliminar y la prueba posterior, se hace notoria una mejoría en conceptos básicos; simultáneamente se hace visible la comprensión 
sobre las representaciones geométricas de las funciones trigonométricas seno, coseno y tangente, en relación a sus respectivas expresiones algebraicas. Al observar los resultados que expresan las matrices generales, se hace evidente un manejo en las características y clasificación de ángulos; se percibe, también, que los educandos distinguen equivalencias y realizan conversiones entre grados sexagesimales y radianes; así mismo se hace explícita una descripción en la relación que existe entre las razones trigonométricas y el triángulo rectángulo. Se trasluce en los estudiantes que hay un perfeccionamiento en la percepción y en la visualización espacial, al describir las representaciones geométricas de las funciones trigonométricas seno, coseno y tangente, relacionándolas con sus respectivas expresiones algebraicas, mientras consolidaba la correspondencia con las variables que causaban cambios en sus caracteres.

En torno al nivel de comprensión que alcanzó cada estudiante, con respecto a los distintos niveles de razonamiento planteados en el modelo propuesto por Van Hiele, sobre la visualización, análisis, clasificación, deducción formal y rigor, se puede señalar que los estudiantes alcanzaron un nivel 4, puesto que, a través del encadenamiento de las guías y construcciones aplicadas, lograron percibir figuras en su totalidad que corresponden al nivel 1, consiguieron describir sus partes y enunciar propiedades de manera informal que hace referencia al nivel 2. Así mismo, en el nivel 3 generalizaron, reconocieron propiedades, enunciaron otras y llegaron a clasificar figuras basándose en ellas; y finalmente, el en nivel 4 lograron comprender las estructura axiomática de las funciones trigonométricas, o sea, consiguieron relacionar directamente la expresión algebraica con la geométrica. No obstante, dicho nivel, que pretende que el estudiante alcance cognitivamente una deducción formal, no se evidencia en su totalidad en dichos estudiantes. Para finalizar, con respecto al nivel 5 de rigor, en el que el estudiante entiende la naturaleza axiomática de las expresiones algebraicas, de las funciones trigonométricas con relación a las representaciones geométricas, los educandos presentaron dificultades para comprender este tipo de demostraciones; por tal razón, se habla de que este nivel no fue alcanzado por ellos.

\section{CONCLUSIÓN}

De los resultados y discusión se destacan las siguientes conclusiones e implicaciones:

Se hizo evidente la necesidad de trabajar con diversas estrategias pedagógicas e instrumentos, con el objetivo del fortalecimiento del proceso de enseñanza-aprendizaje; así como para desarrollar y abordar los métodos educativos que se llevan a cabo, de manera continua, en el aula de clase. De igual forma, es relevante reflexionar sobre la interacción que se da entre docente y estudiante. Amén de buscar diversos métodos que respondan, eficientemente, ante una sociedad que exige cada vez más a sus actores, de tal modo que se debe trabajar diariamente en técnicas que atraigan la atención y la motivación del estudiante en su formación continua y en el que el papel del docente sea exclusivamente de orientador en el proceso de enseñanza.

Este proyecto de investigación, relacionado con las implicaciones pedagógicas de un software de geometría dinámica en la percepción geométrica de las funciones trigonométricas seno, coseno y tangente, develó identificar vacíos conceptuales, relacionados con las nociones básicas: ángulos, ilustraciones de funciones, variaciones y características principales de las funciones trigonométricas. Además, hizo explícita la dificultad que presentaban los estudiantes cuando se les solicitaba que percibieran, visualizaran posibles trayectorias y cambios en los recorridos de los objetos. Por tal razón, se convirtió dicho proceso de abordaje pedagógico en una estrategia significativa, que favoreció la exploración de las representaciones trigonométricas de forma dinámica para el aprendizaje de las funciones trigonométricas. Como valor agregado, las guías bordaban pedagógicamente el uso de un software dinámico en la medida que estimulaba al estudiante a analizar, construir, medir, observar variantes e invariantes y validar situaciones hipotéticas. 
El software dinámico se convierte en una herramienta facilitadora para la comprensión y análisis de las estructuras de las funciones, lo que crea implicaciones en la percepción de la temática, y a su vez, transforma la conducta en el aula de clase, ya que este tipo de herramienta se hace llamativa para ellos, y les erige expectativas, lo que facilita el desarrollo de estructuras mentales, para caracterizar las nociones y formas de las funciones.

Las actividades diseñadas en las guías, con el objeto de mejora del proceso enseñanzaaprendizaje, también apuntan a crear material didáctico que sirva de apoyo al docente y facilite el proceso de aprendizaje de las representaciones geométricas, de las funciones trigonométricas en el aula de clase. De tal manera, que se establecieron una serie de ejercicios que fueron útiles y que coadyuvaron a que el proceso en el aula se convirtiera un acto lúdico e interesante para el estudiante, mientras que desarrollaba operaciones mentales que le permitirán abordar problemas, resolviéndolos a través de la experiencia y expresar su interpretación de lo que había realizado.

Al determinar las implicaciones en el proceso de enseñanza-aprendizaje del software de geometría dinámica en la percepción geométrica de las funciones trigonométricas seno, coseno y tangente, se puede afirmar que el abordaje pedagógico fue pertinente para que los estudiantes obtuvieran la capacidad de, entre otras cosas: reconocer, analizar y caracterizar funciones y manejar un lenguaje técnico. Conjuntamente, se ratificó el desarrollo del nivel de razonamiento a otro nivel de orden superior para alcanzar el pensamiento geométrico. De igual manera, es de resaltar que dicha investigación fue una experiencia significativa para continuar fortaleciendo proyectos que inviten a los docentes a cuestionarse sobre su quehacer pedagógico en la enseñanza de las matemáticas.

Para concluir, es relevante resaltar que en el desarrollo de la investigación no se consideraron aspectos cualitativos, actitudinales ni funciones trigonométricas inversas, a raíz del tiempo que conlleva el abordar dichos elementos, sin embargo los resultados arrojados en el proyecto se convierten en el abrebocas para futuros trabajos investigativos, lo que dará continuidad a la tarea y compromiso de formar integralmente a los estudiantes.

\section{REFERENCIAS BIBLIOGRÁFICAS}

Acosta, M. E. (2005). Geometría experimental con Cabri: una nueva praxeología matemática. Educación Matemática, 17(3), 121-140. Recuperado el 12/01/2015, en: http://www.redalyc.org/ pdf/405/40517307.pdf

Ausubel, D., Novak, J. y Hanesian, H. H. (2001). Psicología educativa. Un punto cognoscitivo. México D. F: Trtillas.

Ballester, A. (2002). El aprendizaje significativo en la práctica. Recuperado el 12/01/2015, en: http:// www.aprendizajesignificativo.es/mats/El_aprendizaje_significativo_en_la_practica.pdf

Cantoral, R. y Farfan, R. (2004). Desarrollo conceptual del cálculo. México D. F., México. Thomson.

D’Amore, B. (2006). Didáctica de la matemática. Bogotá, Colombia: Cooperativa editorial magisterio.

Fouz, F. (s.f.). Modelo de Van Hiele para la didáctica de la geometría. Recuperado el 15/09/2014/, en: http://www.xtec.cat/ rnolla/Sangaku/SangWEB/ PDF/PG-04-05-fouz.pdf

Carulla, C. y Gómez, P. (s.f.). Enseñanza constructivista, conocimiento didáctico del profesor y análisis didáctico en matemáticas. El caso de la función cuadrática. Recuperado el 15/09/2014/, en: http:// funes.uniandes.edu.co/365/1/GomezP01-2585. PDF

Gutiérrez, A. y Jaime, A. (1998). On the assessment of the Van Hile levels of reasoning. Focus on learning problems in mathematics, 20 (2-3), 2746. Recuperado el 15/09/2014/, en: http://www. uv.es/ gutierre/archivos1/textospdf/GutJai98.pdf

Hernández, R., Fernández C. y Baptista, M. P. (2010). Metodología de la investigación. México D.F., México: McGraw-Hill.

Rizzolo, S. (s.f.). Diseño de actividades geométricas interactivas en el marco conceptual del modelo de Van Hiele. Recuperado el 15/09/2014/, en: https://dl.dropboxusercontent.com/u/44162055/ didact_mate $/ 5 . \% 20 \% 20$ Dise \% C3 \% B1 o \% 20 de $\% 20$ Actividades $\% 20$ Geom $\%$ C3 \% A9tricas $\% 20$ Interactivas $\% 20$ en $\% 20$ el $\% 20$ Marco $\% 20$ Conceptual\%20del \% 20Modelo \%20de \%20Van \% 20 Hiele.pdf 
Sánchez, A. A. (2010). Estrategias didácticas para el aprendizaje de los contenidos de trigonometría empleando las TICs. Edutec, (31) Recuperado el 15/09/2014/, en: http://edutec.rediris.es/ Revelec2/revelec31/articulos_n31_pdf/Edutec-e_ n31_Sanchez.pdf

Sierra, M. (2011). Investigación en educación matemática: objetivos, cambios, criterios, método y difusión. Educatio Siglo XXI, 29 (2), 173-198 Recuperado el 15/09/2014/, en: http:// digitum.um.es/xmlui/bitstream/10201/27206/1/ Investiga ci \% C $3 \%$ B 3 n \% 20 e n \% 20 Educaci \% C3 \% B3n \% 20Matem \% C3\% A1tica \% 20 objetivos $\% 2$ c $\% 20$ cambios $\% 2$ c $\% 20$ criterios \% 2c \% 20m \% C3 \% A 9todo \% 20y \% 20 difusi $\%$ C3\%B3n.pdf
Soto, M. (1993). Didáctica de las Matemáticas. Revista de la Facultad de Educación Universidad de Castilla, (8), 173-192. Recuperado el 15/09/2014/, en: http://dialnet.unirioja.es/servlet/ articulo? codigo $=2282535$

Van Hiele, P. M. (1990). El problema de la comprensión en conexión con la comprensión de los escolares en el aprendizaje de la geometría. Recuperado el 15/09/2014/, en: http://www.uv.es/ aprengeom/archivos2/VanHiele57.pdf

Villalobos, E. M. (2006). Didáctica integral y el proceso de aprendizaje. México D. F.: Trillas. 